\title{
ETIOLOGY OF GASTROINTESTINAL BLEEDING IN CHILDREN
}

\author{
Nicoleta Gimiga, Smaranda Diaconescu, Claudia Olaru, Alexandru Olaru, \\ Gabriela Paduraru, Ancuta Ignat, Marin Burlea \\ „, Gr. T. Popa” University of Medicine and Pharmacy, Iasi
}

\begin{abstract}
Objectives. Identification of clinical, endoscopic, etiological characteristic of children diagnosed with upper and lower gastrointestinal bleeding.

Material and methods. It was conducted a descriptive retrospective study over a 3 year period (January 2010 to December 2012) on 107 children aged 1-18 years hospitalized for gastrointestinal bleeding in "St. Mary" Children's Emergency Hospital, lasi. The study group does not include gastrointestinal bleeding from surgical emergencies, infectious diseases, intestinal diseases with immunological or toxic mechanism. Individualized retrospective analysis included historical data, clinical, endoscopic and histological targeted for etiologic diagnosis of gastrointestinal bleeding. All patients were investigated by upper gastrointestinal endoscopy/colonoscopy after the procedure was explained and informed consent was obtained.

Results. From the batch of 107 children, 39 (36.4\%) presented with upper gastrointestinal bleeding (UGIB) 6 $(5.1 \%)$ was variceal, non-variceal in $33(94.4 \%)$ cases, and $68(63.5 \%)$ presented with lower gastrointestinal bleeding (LGIB) The main etiologycal aspect of UGB was erosive gastritis $30.8 \%$, esophagitis in $15.4 \%$, duodenitis in $15.4 \%$, gastric and duodenal ulcers $5,1 \%$ and respectivily $10.3 \%$ of cases, Mallory-Weiss syndrome in $2.6 \%$, multiple etiology in 10 cases $15.4 \%$. Causes of LGIB were colorectal polyps in $41.2 . \%$, ulcerative colitis $20.6 \%$, non specific lessions in $17.6 \%$ anal fissures $13.2 \%$, intestinal polyposis $4.4 \%$,rectal diverticula $1.5 \%$ and vascular malformations $1.5 \%$. It was practiced concomitent endoscopic surgery for rectal polyps.

Conclusions. Lower gastrointestinal bleeding was the most common causes related to minor conditions: colorectal polyps, anal fissures, nonspecific lesions.

Non-variceal gastrointestinal bleeding the most common form associated with erosive gastritis, esophagitis, duodenal ulcer, gastric ulcer.

Endoscopy proved to be a useful investigation in the diagnosis of gastrointestinal bleeding and a therapeutic useful tool in certain cases.
\end{abstract}

Keywords: gastrointestinal bleeding, children, etiology, endoscopy, colonoscopy

\section{INTRODUCTION}

Gastrointestinal bleeding remains one of the largest, frequent and important emergency of pediatric gastroenterology, in terms of both diagnostic and therapeutic approach.

The incidence of digestive haemorrhage is not very well established, in Europe being 0,4-1,6\% for upper gastrointestinal bleeding (1) while the lower gastrointestinal haemorrhage totalize $0.3 \%$ of emergency presentations. (2)

In paediatric practice there are described various etiological aspects, from benign conditions which require no treatment to severe and potential lethal etiologies that require immediate intervention.

\section{OBJECTIVES}

Identification of clinical, endoscopic and etiological characteristics of children diagnosed with upper and lower gastrointestinal bleeding.

\section{MATERIAL AND METHOD}

We conducted a descriptive retrospective study over a period of 3 years (January 2010 to December

Corresponding author:

Smaranda Diaconescu, „Gr.T. Popa” University of Medicine and Pharmacy, 16 Universității Street, lasi

E-mail: turti23@yahoo.com 
2012). We investigated 107 children aged between 1-18 years, which were addressed for gastrointestinal bleeding to St. Mary Clinical Emergency Hospital in order to determine the etiologies of gastrointestinal bleeding with the following reasons: hematemesis, melena, recthorragia, hematochezia, unexplained anaemia objectified by at least 2 positive fecal blood tests.

The study group does not include gastrointestinal bleeding from surgical emergencies, infectious diseases, intestinal diseases with immunological or toxic mechanism, patients who ingested substances that may be confused with false hemorrhage (fruits and vegetables containing peroxidase: broccoli, radishes, tomatoes, broth beets, blueberries, drinks containing red dye, iron supplements, bismuth salts). (3)

The data were collected from observation charts of patients and endoscopy reports.

All children were investigated by endoscopy/ colonoscopy/rectosigmoidoscopy after the technique was explained and the informed consent was obtained. Patients were under general anesthesia with propofol or midazolam sedation depending on age.

The batch of patients was divided into 2 subgroups, 39 patients with upper gastrointestinal bleeding and 68 patients with lower gastrointestinal bleeding.

Biopsies were performed under direct visualization of the mucosa and for $\mathrm{H}$. pylori detection were used mucosa specimens: rapid urease test, microscopic examination and culture of biopsy material.

Statistical analysis and graphic representations were performed using SPSS 20.

\section{RESULTS}

We included in the study 107 patients with documented upper and lower gastrointestinal bleeding who were addressed to our clinic during the study period of time.

\section{Subgroup of upper gastrointestinal bleeding.}

The subgroup of patients with upper gastrointestinal bleeding totalized 39 patients, aged between $1-18$ years, mean age $9.56 \pm 5.38$ years. From these $56.4 \%$ were male and $43.6 \%$ were girls. The main reason for presentation was hematemesis $(46.2 \%)$, followed by melena (35.9\%) while 7 patients $(17.9 \%)$ were presented with both symptoms, hematemesis and melena. (Fig. 1)

$20.5 \%$ of patients in the study group had history of non antiinflamatory drugs (NSAIDs) consump-

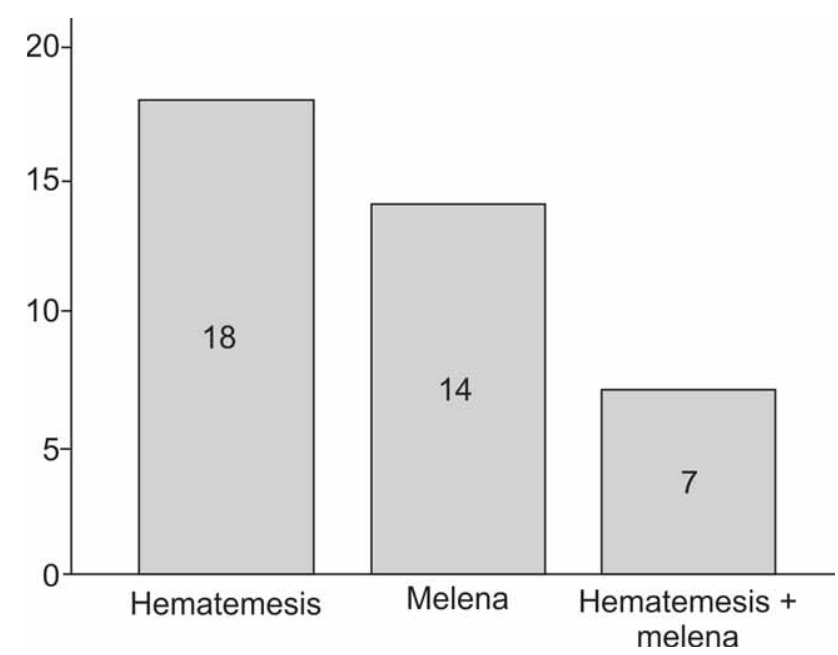

FIGURE 1. Presenting symptoms for the subgroup of upper gastrointestinal bleeding

tions, such patients having more frequent in aetiology pathology such erosive gastritis, gastric ulcer, esophagitis. (Fig. 2)

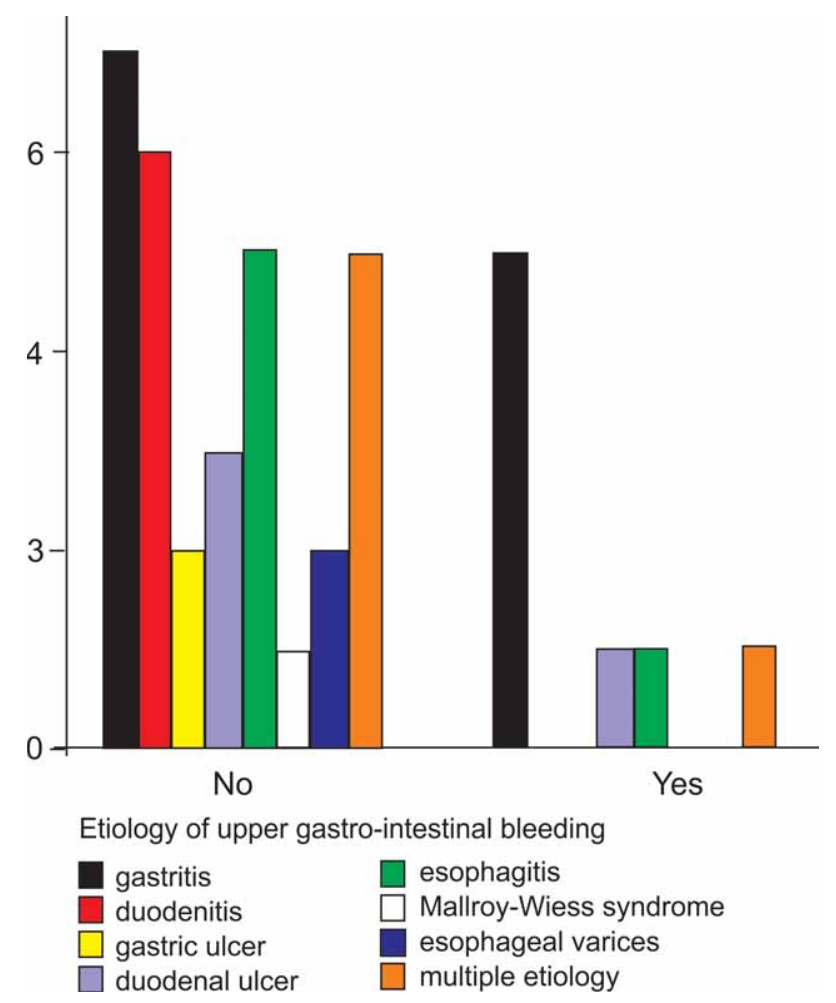

FIGURE 2. Non antiiflamatory drugs intake for the subgroup of upper gastrointestinal bleeding

More than half $(51.3 \%)$ of patients in the study had gastric infection with $\mathrm{H}$. pylori. In these patients frequently prevailed duodenitis and duodenal ulcer. (Fig. 3)

The most common causes of upper gastrointestinal bleeding observed in the studied subgroup was erosive gastritis, occurred at $30.8 \%$ of patients. 


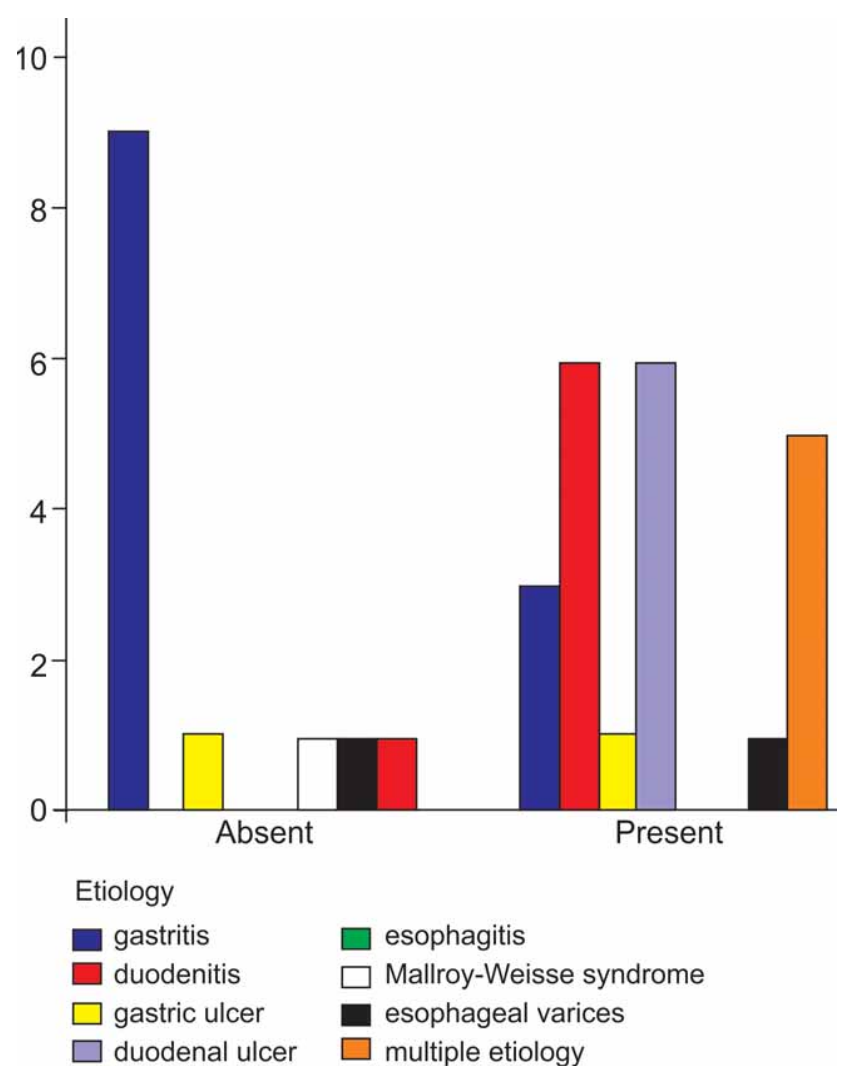

FIGURE 3. The presence of $H$. pylori infection and corelation with etiology of upper gastrointestinal bleeding

It was followed by esophagitis (15.4\%), duodenitis $(15.4 \%)$, duodenal ulcer $(10.3 \%)$, gastric ulcer (5.1\%), oesophageal varices (5.1\%), Mallory-
Weiss syndrome (2.6\%), multiple etiologies $15.4 \%$ (gastritis and duodenitis, esophagitis and gastritis, gastritis and duodenal ulcers, esophagitis and gastric ulcer, gastritis and gastric polyps, gastric and duodenal ulcer). (Fig. 4)

Both gender distribution male and female, the main etiological factor of upper gastrointestinal bleeding was gastritis $(27.3 \%$ boys and $23.5 \%$ girls), followed by etiologies as esophagitis and duodenitis (by $22.7 \%$ each) for males and duodenal ulcer and duodenitis (17.6\% and 5.9\%) for females.

\section{Subgroup of lower gastrointestinal bleeding}

The subgroup of patients with lower gastrointestinal bleeding consisted in 68 patients, aged 1-18 years, mean age $7.01 \pm 5.09$ years. From these $54.4 \%$ were male and $45.6 \%$ were girls

The most common reason for presentation was hematochezia $(54.4 \%)$, followed by recthoragia $38.2 \%$, while $7.4 \%$ had a positive test for occult bleeding. (Fig. 5)

The most common cause of lower gastrointestinal bleeding was colorectal polyp, $41.2 \%$, polyps were sized between $0.5-1.5 \mathrm{~cm}$. The polyps were extracted in the same colonoscopy session. The second causes was ulcerative colitis, $20.6 \%$, followed by non-specific lesions $17.6 \%$ such as localized or disseminated inflamation, erythema, decreased or increased vascular marking and fissure

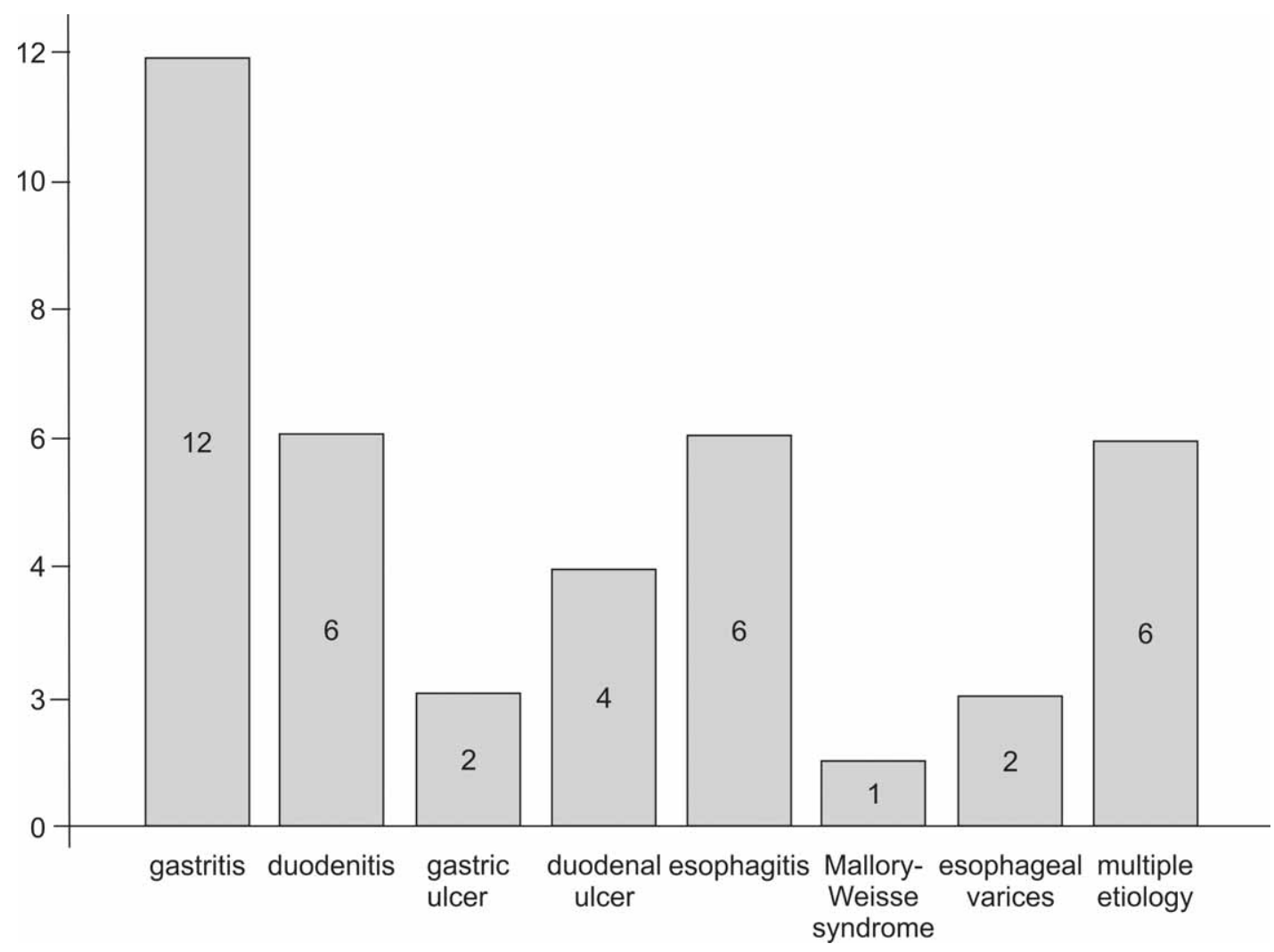

FIGURE 4. Etiology of upper gastrointestinal bleeding 


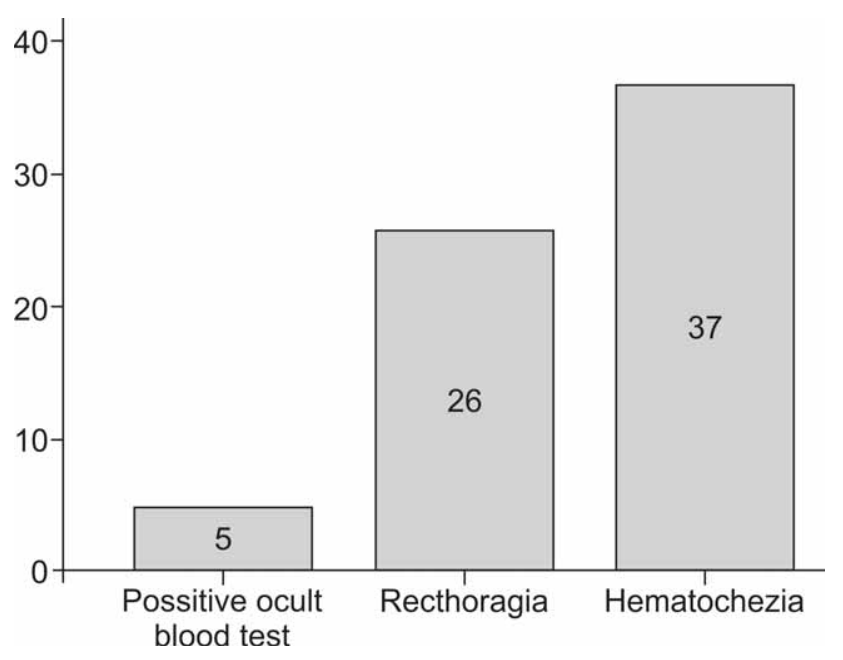

FIGURE 5. Presenting symptoms for the subgroup of lower gastrointestinal bleeding

(13.2\%), intestinal polyposis $(4.4 \%, 1$ case of Peutz-Jeghers syndrome 1 case of Bannayan-Ryley syndrome, 1 case of Gardner syndrome), rectal diverticula $(1.5 \%)$ and vascular malformations (1.5\%). (Fig. 6) Colorectal polyps were $0.5-1.5 \mathrm{~cm}$ in diameter, the vast majority were located on recto-sigmoid, all polyps were extracted in the same seasionof colonoscopy and the juvenile polyp was the most trequenthistological type.

Gender distribution of etiologies, in both boys and girls it was found that the main etiological aspect was colorectal polyp found in $45.9 \%$ and $35.5 \%$ respectively.

\section{DISCUSSIONS}

Gastrointestinal bleeding is an alarming event among children and parents, and an important reason for presentation to the hospital. Often the gastrointestinal haemorrhage is self-limited and does not cause hemodynamic compromise but the addressability to medical service is quite high for diagnosis and treatment, as demonstrated by this study where we found anal fissures to be an etiologic agent in a higher percent of lower gastrointestinal bleeding. Previous studies have shown that rectal bleeding in children is often a self-limited condition that requires no treatment but only supportive measures. $(4,5)$

In both subgroups of upper gastrointestinal bleeding and lower gastrointestinal bleeding, sex distribution shows a predominance of males but no statistically significant differences $(\mathrm{p}<0.1)$, as demonstrated in other studies. $(6,7)$ Male predominance was attributed for some authors to the protective effect of estrogen on gastric and duodenal injury, argument demonstrated by Smith et al. (8)

Hematemesis was the most important symptom of presentation of upper gastrointestinal bleeding accounting for $46.2 \%$, followed by melena $35.9 \%$, similar results were found in other studies conducted by El. Mouzan (50.0\%) and Huang IF (68.8\%). This is explained by the fact that hematemesis is an alarming event for the patient and family than melena that is observed later, leading to a delayed of presentation to health services. $(9,10)$

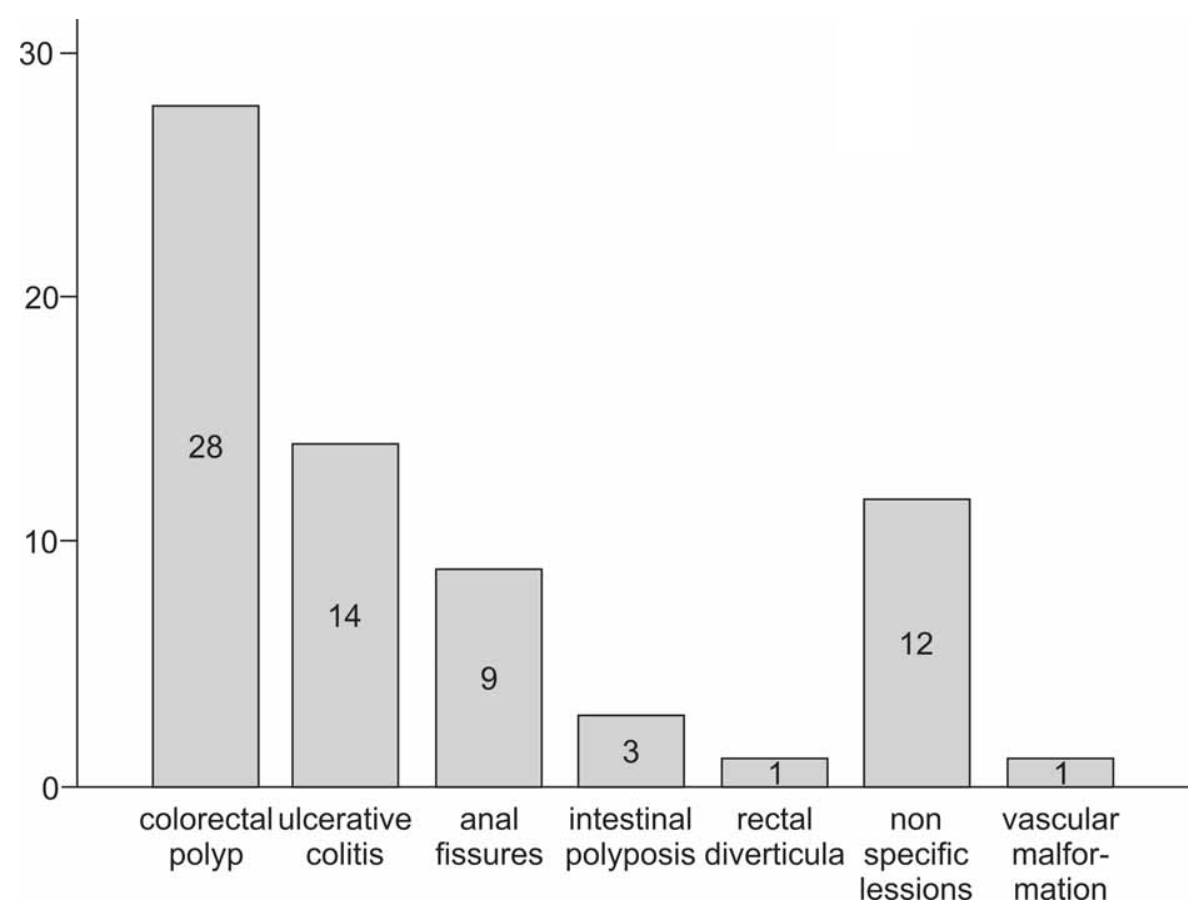

FIGURE 6. Etiology of lower gastrointestinal bleeding 
Hematochezia was the most common symptom presentation for lower gastrointestinal bleeding followed by recthoragia, similar data reported in studies conducted by Hossein et al. (11)

The main aetiology for upper gastrointestinal bleeding was erosive gastritis representing approximately $30.8 \%$ of patients, similar data were found in studies conducted by EL Mouzan (44.0\%) and Huang IF (44.6\%). (9.10) Variceal bleeding represented $5.1 \%$, a small percentage, the explanation for this may be that we are not an active hepatology unit but rather a unit that provides gastroenterology services. In developed countries variceal bleeding occurs in higher percentages, as demonstrated by studies conducted by Abdullah et al, 39\% and Quak SH $(23.4 \%)$. (12,13) A second cause of upper gastrointestinal bleeding was erosive esophagitis $15.4 \%$, esophagitis is generally secondary to gastro-esophageal reflux, similar data were found in studies conducted by Mandana et al. (14)

Gastric and duodenal ulcers were found in 5.3\% and $10.1 \%$, results comparable with other studies conducted by El Mouzan (8.5\% vs 6.8\%) and SK Mittal (1.3\% vs 0.4\%). (9.15)

8 patients $(20.5 \%)$ had a history of drug intake, 6 ingested NSAIDs for a short period of time for acute respiratory infections and 2 patients had ingested steroids for conditions such as juvenile chronic arthritis, nephrotic syndrome, comparable data were found in research conducted by Mittal and El Mouzan. $(9,15)$

The correlation between $H$. pylori infection and upper gastrointestinal bleeding was suggested by the presence of this infections in $51.3 \%$ of patients. In Romania the prevalence of $H$. pylori infection is $36.65 \%$ (Slavescu et al 2012) higher incidence of infection by $H$. pylori occurs in countries with a higher prevalence of gastric ulcer. $(7,16)$

Regarding lower gastrointestinal haemorrhage the main aetiology was colorectal polyp representing $41.2 \%$, followed by ulcerative colitis, nonspecific lesions and anal fissures. Comparing our find- ings with similar studies regarding etiological aspects of lower gastrointestinal bleeding we notice that they do not greatly differ. The most common cause of lower gastrointestinal bleeding in the Middle East, Western countries and the Chinese population was -colorectal polyp (5, 17-18) In Egyptian population the main cause of lower gastrointestinal bleeding is infectious colitis followed by colorectal polyp. (19)The most common form of polyps were usually solitary juvenile polyps, we had only 3 cases of intestinal polyposis where we found polyps sized between $0.5-2 \mathrm{~cm}$.

Anal fissures are an important etiologic factor accounting for $13.2 \%$, fissures usually occur secondary to chronic constipation a disease that is more common in toddlers and schoolchildren. This issue should be investigated in future studies to elucidate the favoring conditions or the substrate of this pathology.

In our study, ulcerative colitis represents $20.6 \%$, doing a comparison with other studies performed in Western populations (20) we notice that is a quite important percentage and also a warning signal that requires further investigation.

\section{CONCLUSIONS}

Lower gastrointestinal bleeding was the most common causes related to minor conditions: colorectal polyps, anal fissures, nonspecific lesions.

Non-variceal gastrointestinal bleeding the most common form associated with erosive gastritis, esophagitis, duodenal ulcer, gastric ulcer.

Endoscopy proved to be a useful investigation in the diagnosis of gastrointestinal bleeding and a therapeutic useful tool in certain cases

\section{Acknowledgment}

We thank to all the staff of Endoscopy Unit and Pathology Department, without their support this work would not have been possible.

\section{REFERENCES}

1. Lacroix J., Nadeau D., Laberge S. et al. Frequency of upper gastrointestinal bleeding in a pediatric intensive care unit. Crit. Care Med1992; 20:35-42

2. Pant C., Sankararaman S., Deshpande A. et al. Gastrointestinal bleeding in hospitalized children in the United States. Curr Med Res Opin. 2014; 30(6):1065-9.

3. Karjoo S., Liacouras C.A. Lower gastrointestinal bleeding. In: Schwartzs et al. Clinical handbook of pediatrics, 5th ed. Eds. Wolters Kluwer Health/Lippincott Williams \& Wilkins, Philadelphia 2013:368.
4. Boyle J.T. Gastrointestinal bleeding in infants and children. Pediatr Rev 2008; 29:39-52.

5. Zahmatkeshan M., Fallahzadeh E., Najib K.S. Etiology of Lower Gastrointestinal Bleeding in Children:A Single Center Experience from Southern Iran. Middle East J Dig Dis 2012; 4:216-3.

6. Motamed F., Najafi M., Fallahi M. et al. Colonoscopic Findings In Children With Lower Gastrointestinal Bleeding. Govaresh 2008; 13(1):54-57 
7. Houben C.H., Chiu P.W., Lau J.Y., et al. Duodenal ulcers dominate acute upper gastrointestinal tract bleeding in childhood: a 10-year experience from Hong Kong. J Dig Dis. 2008; 9:199-203

8. Smith A., Contreras C., Ko K.H. et al. Gender-specific protection of estrogen against gastric acid-induced duodenal injury: stimulation of duodenal mucosal bicarbonate secretion.Endocrinology 2008; 149:4554-4566

9. E.L. Mouzan M.I., Abdullah A.M., A.I. Mofleh I.A. Yield of endoscopy in children with hematemesis. Trop Gastroenterol 2004; 25: 44-46

10. Huang I.F., Wu T.C., Wang K.S. et al. Upper gastrointestinal endoscopy in children with upper gastrointestinal bleeding. $J$ Chin Med Assoc 2003; 66:271-275

11. Hossein M., Dehghani S.M., Nikzadeh H., Malekpour A. Lower Gastrointestinal Bleeding in Children: Experiences From Referral Center in Southern Iran. J Compr Ped 2013; 3(3):115-18

12. Quak S.H., Lam S.K., Low P.S. Upper gastrointestinal endoscopy in children. Singapore Med J 1990; 31(2):123-6

13. Abdullah J., Afrah A., A.I. Maeeni et al. Upper Gastrointestinal Bleeding In Children. J Fac Med Baghdad. 2012; 54(3):223-227

14. Mandana R., Shoaran M., Majidy H. Diagnostic Endoscopy and Clinical Characteristics of Gastrointestinal Bleeding in Children: a
10-Year Retrospective Study, Iran Red Crescent Med J 2013; 15(9):794-7

15. Mittal S.K. Karla K.K. Aggarwal V. Diagnostic GI endoscopy for hematemesis in children: experience from a pediatric gastroenterology center in north India. Indian J Pediatr. 1994; 61(6):651-4

16. Slăvescu K, Sarban C., Pîrvan A. et al. Prevalence of Helicobacter pylori infection in children with gastritis and peptic ulcer disease in north-western and central Romania, Clujul Medical 2012; 85(3): 457-462

17. Durno C.A. Colonic polyps in children and adolescents. Can J Gastroenterol 2007:21(4):233-9.

18. Yu B., Jun P., Jun G. et al. Epidemiology of lower gastro-intestinal bleeding in China: Single-center series and systematic analysis of Chinese literature with 53951 patients. J Gastro and Hepat 2010; 26:678-8.

19. El-Khayat H.A., El-Hodhod M.A., Abd El-Basset, F.Z. et al. Rectal bleeding in Egyptian children. Ann Trop Pediatr 2006; 26:337-44

20. Fox V.L. Gastrointestinal bleeding in infancy and childhood. Gastroenterol Clin North Am 2000; 29:70107-2.2000. 